Pacific Journal of Mathematics

SOME REPRESENTATIONS OF THE AUTOMORPHISM GROUP
OF AN INFINITE CONTINUOUS HOMOGENEOUS MEASURE

ARTHUR LARRY LIEBERMAN 


\title{
SOME REPRESENTATIONS OF THE AUTOMORPHISM GROUP OF AN INFINITE CONTINUOUS HOMOGENEOUS MEASURE ALGEBRA
}

\author{
ARTHUR LIEBERMaN
}

\begin{abstract}
Let $(X, \tau, m)$ be an infinite continuous homogeneous measure space. Let $A$ be the measure algebra of $(X, \tau, m)$ and $G$ be the automorphism group of $A$. The canonical representation of $G$ on the subspace of all elements of $\otimes^{n} L^{2}(X, \tau, m)$ of some fixed maximal symmetry type is irreducible. Two such representations are equivalent iff they correspond to the same $n \in N$ and to the same partition of $n$.
\end{abstract}

1. Introdution and notation. Let $H$ be a Hilbert space. If $n \in N$, let $\otimes^{n} H$ denote the tensor product of $H$ with itself $n$ times. Let $S_{n}$ be the symmetric group on the first $n$ natural numbers. Let $\theta$ be the unique representation of $S_{n}$ on $\otimes^{n} H$ such that $\theta(g)\left(v_{1} \otimes v_{2} \otimes\right.$ $\left.\cdots \otimes v_{n}\right)=v_{g(1)} \otimes v_{g(2)} \otimes \cdots \otimes v_{g(n)}$ if $g \in S_{n}$ and $v_{i} \in H$ for $1 \leqq i \leqq n$.

If $S$ is a set of operators on a Hilbert space and $S$ contains the adjoint of each of its members, then the commutant $S^{\prime}$ of $S$ is a von Neumann algebra; the double commutant $S^{\prime \prime}$ of $S$ is the smallest von Neumann algebra which contains $S$ [1].

$\theta\left(S_{n}\right)^{\prime \prime}$ is generated by its mutually orthogonal minimal projections [9]. These projections are in a 1-1 correspondence with the maximal symmetry types. The vectors in the range of a minimal projection are said to be of the corresponding maximal symmetry type.

Let $U(H)$ be the unitary group on $H$. Let $I$ be the canonical representation of $U(H)$ on $H$. If $n \in N$, let $\otimes^{n} I$ be the tensor product of $I$ with itself $n$ times. The restriction of $\otimes^{n} I$ to the subspace of all vectors of any fixed maximal symmetry type is irreducible. If $H$ is finite dimensional, this result is classical [9]; if $H$ is infinite dimensional, it is due to Segal [8]. The author has obtained similar results [3] for the symmetric groups on an infinite set $S$, where $S$ is an orthonormal basis for $H$. In all three cases, these representations can be explicitly characterized.

Below we prove that the analogous representations of the automorphism group of an infinite homogeneous measure algebra are irreducible. No characterization of these representations is known.

Definition. A continuous measure algebra $\langle M, \mu\rangle$ is homogeneous if and only if two principal ideals are isomorphic whenever their generators have equal and finite measure. A continuous measure space is homogeneous if and only if its measure algebra is homogeneous. 
These definitions differ slightly from those of Maharam [4]. Note that we have no $\sigma$-finiteness requirement.

For the remainder of this paper, let $(X, \tau, m)$ be an infinite homogeneous measure space with measure algebra $\langle A, m\rangle$; Let $G$ be the automorphism group of $\langle A, m\rangle$. Let $H=L^{2}(X, \tau, m)$. Let $R$ be the canonical representation of $G$ on $H ; R(g) f=f g^{-1}$, where $g \in G$ and $f \in H$. If $n \in N, \otimes^{n} R$ will denote the tensor product or $R$ with itself $n$ times.

The remainder of this paper is devoted to proving the following theorem:

THEOREM 1. Let $(X, \tau, m)$ be an infinite continuous homogeneous measure space with measure algebra $\langle A, m\rangle$. Let $G$ be the automorphism group of $\langle A, m\rangle$. Let $n \in N$, and let $y$ denote a maximal symmetry type. Let $H_{n y}$ be the subspace of $\otimes^{n} H$ of all vectors of maximal symmetry type $y$. If $g \in G$, let $R_{n y}(g)=\otimes^{n} R(g) \mid H_{n y}$. Then $R_{n y}$ is an irreducible representation of $G$.

Let $m \in N$ and let $k$ be a maximal symmetry type. Then $R_{n y} \cong R_{m k}$ if and only if $n=m$ and $y$ corresponds to the same partition of $n$ as $k$ does.

2. Proof of the theorem. The proof of the theorem requires two definitions and two lemmas.

Definition. A decomposition of $(X, \tau, m)$ of type $r$, where $r$ is a positive real number, is a subset $D=\left\{E_{\lambda}: \lambda \in \Lambda\right\}$ of $\tau$, where $\Lambda$ is an index set, such that $X=\bigcup_{\lambda_{\in A}} E_{\lambda}, m\left(E_{\lambda}\right)=r$ if $\lambda \in \Lambda$, and $E_{\lambda_{1}} \cap E_{\lambda_{2}}=\phi$ if $\lambda_{1}, \lambda_{2} \in \Lambda$ and $\lambda_{1} \neq \lambda_{2}$.

Definition. Let $D_{1}=\left\{E_{\lambda}: \lambda \in \Lambda\right\}$ and $D_{2}=\left\{F_{j}: \delta \in \Delta\right\}$ be decompositions of $(X, \tau, m)$, where $\Lambda$ and $\Delta$ are index sets. We say that $D_{1}$ is subordinate to $D_{2}$ if every member of $D_{1}$ is a subset of a member of $D_{2}$.

Lemma 1. Let $J$ be a Hilbert space. Let $\Gamma$ be a unitary group on $J_{0}$ Let $P$ be a projection on $J$. Let $\left\{P_{q}: q \in Q\right\}$ and $\left\{P_{q}^{1}: q \in Q\right\}$, where $Q$ is an index set, be sets of projections on $J$. Assume:

(1) $P J \cap P_{q}^{1} J \neq 0$ if $q \in Q$.

(2) $P_{q} J \cap P_{q}^{1} J \neq 0$ if $q \in Q$.

(3) $P \in \Gamma^{\prime \prime}$.

(4) $P_{q} \in \Gamma^{\prime \prime}$ and $P_{q}^{1} \in \Gamma^{\prime \prime}$ if $q \in Q$.

(5) $I=\operatorname{lub}\left\{P_{q}: q \in Q\right\}$.

(6) $\left\{P \gamma P \mid P J: \gamma \in \Gamma^{\prime \prime}\right\}$ acts irreducibly on $P J$. 
(7) $\left\{P_{q} \gamma P_{q} \mid P_{q} J: \gamma \in \Gamma^{\prime \prime}\right\}$ acts irreducibly on $P_{q} J$ if $q \in Q$.

(8) $\left\{P_{q}^{1} \gamma P_{q}^{1} \mid P_{q}^{1} J: \gamma \in \Gamma^{\prime \prime}\right\}$ acts irreducibly on $P_{q}^{1} J$ if $q \in Q$.

Then $\Gamma$ acts irreducibly on $J$.

Proof. Let $T \in \Gamma^{\prime}$. Then $T \mid P J=c I$ for some scalar $c$ by Assumptions 3 and 6. Let $q \in Q$. By 4 and $8, T \mid P_{q}^{1} J=c_{q}^{1} I$ for some scalar $c_{q}^{1}$. By $1, c_{q}^{1}=c$. By 4 and $7, T \mid P_{q} J=c_{q} I$ for some scalar $c_{q}$. By 2, $c_{q}=c_{q}^{1}=c$. Therefore $T=c I$ on the subspaced spanned by $\mathrm{U}_{q \in Q}\left(P_{q} J\right)$. By 5 , this subspace is dense in $J$.

LEMma 2. Let $r$ be a positive real number. Let $D$ be a decomposition of $(X, \tau, m)$ of type $r$. Let $E_{i} \in D$ for $1 \leqq i \leqq n$. Assume $E_{i} \neq E_{j}$ if $1 \leqq i, j \leqq n$ and $i \neq j$. Let $Y$ be projection onto the subspace of $\otimes^{n} H$ spanned by $\theta\left(S_{n}\right)\left[C\left(E_{1}\right) \otimes C\left(E_{2}\right) \otimes \cdots \otimes C\left(E_{n}\right)\right] \cdot \quad\left(C\left(E_{i}\right)\right.$ is the characteristic function of $E_{i}$.) Then $Y \in\left(\left(\otimes^{n} R\right)(G)\right)^{\prime \prime}$.

Proof. Let $M=\{1,2, \cdots, n\}$ and $2^{M}$ be the power set of $M$. If $s \in 2^{M}$, let $G_{s}=\left\{g \in G: g\left(E_{i}\right)=E_{i}\right.$ for $\left.i \in s\right\}$. Let $P_{s}$ be projection onto $\left\{v \in \bigotimes^{n} H:\left(\otimes^{n} R\right)(g) v=v\right.$ for all $\left.g \in G_{s}\right\}$. Then $P_{s} \in\left(\left(\bigotimes^{n} R\right)\left(G_{s}\right)\right)^{\prime \prime}$ $\subseteq\left(\left(\otimes^{n} R\right)(G)\right)^{\prime \prime}$ by the double commutant theorem [1].

Homogeneity of a measure algebra implies that two principle ideals whose generators have equal and finite measure are carried onto one another by an automorphism of the algebra. To prove this, let $x$ and $y$ be generators of the principle ideals $\bar{x}$ and $\bar{y}$. Assume $m(x)=m(y)<\infty$. Let $x_{1}=x-y$ and $y_{1}=y-x$. By homogeneity there is an isomorphism $\delta$ of $\bar{x}_{1}$ onto $\bar{y}_{1}$. If $E \in A$, let $\Delta(E)=$ $\left(E \cap \widetilde{\left(x_{1} \cup y_{1}\right)}\right) \cup \delta\left(E \cap x_{1}\right) \cup \delta^{-1}\left(E \cap y_{1}\right) . \quad \Delta$ is an isomorphism of $A$ and $\Delta(\bar{x})=\bar{y}$.

Homogeneity of a measure space implies that any measurable function on the space which is invariant under the automorphism group of the corresponding measure algebra must be a constant a.e. Consequently, $P_{s}$ is projection on the subspace of $\otimes^{n} H$ spanned by vectors of the form $f_{1} \otimes f_{2} \otimes \cdots \otimes f_{n}$, where for each $j, 1 \leqq j \leqq n, f_{j}=$ $C\left(E_{i}\right)$ for some $i \in s$.

$Y=P_{M} I_{s \in 2 M-M}\left(I-P_{s}\right)$. (Note that the operators in this product all commute.) This follows from the description of the $P_{s}$ and an easy consideration of various cases.

Proof of Theorem 1. Let $D$ be a decomposition of $(X, \tau, m)$. If $Z$ is a subset of $D$ of cardinality $n$, let $Y(Z)$ be the projection associated with $Z$ as in Lemma 2 and let $T(Z)$ be projection on $Y(Z) H_{n y}$. By Lemma 2, $Y(Z) \in\left(\otimes^{n} R(G)\right)^{\prime \prime}$; consequently, $T(Z) \in R_{n y}(G)^{\prime \prime}$. Let $T_{n}(D)=\otimes T(D)$, where the sum is taken over all $Z \cong D$ such that 
$Z$ has $n$ members; $T_{n}(D) \in R_{n y}(G)^{\prime \prime}$. Let $P_{n}(D)=\operatorname{lub}\left\{T_{n}\left(D^{\prime}\right): D^{\prime}\right.$ is subordinate to $D\} ; P_{n}(D) \in R_{n y}(G)^{\prime \prime}$.

Select a decomposition $D$ of type 1 . In Lemma 1 , let $J=H_{n y}, \Gamma=$ $R_{n y}(G)$, and $P=P_{n}(D)$. Let $Q$ be the set of decompositions of $(X, \tau, m)$ of type 1. If $d \in Q$, let $\alpha(d)$ be any member of $Q$ such that $\exists F_{1} \in \alpha(d), F_{2} \in d$, and $F_{3} \in D$ such that $m\left(F_{1} \cap F_{2}\right)>0$ and $m\left(F_{1} \cap F_{3}\right)>0$. Let $P_{d}=P_{n}(d)$ and $P_{d}^{1}=P_{n}(\alpha(d))$.

The condition $m\left(F_{1} \cap F_{2}\right)>0$ leads to $P_{d} J \cap P_{d}^{1} J \neq 0$; this is Hypothesis 2 of Lemma 1 . To see this, let $E_{i}, 1 \leqq i \leqq n$, be chosen so that $E_{i} \subseteq\left(F_{1} \cap F_{2}\right), m\left(E_{i}\right)>0$, and $E_{i} \cap E_{j}=\phi$ if $i \neq j$. Let $v=C\left(E_{1}\right) \otimes C\left(E_{2}\right) \otimes \cdots \otimes C\left(E_{n}\right)$. Let $w$ be the projection of $v$ onto $H_{n y}$. Then $w \neq 0, w \in P_{d} J$, and $w \in P_{d}^{1} J$. Similarly, the condition $m\left(F_{1} \cap F_{3}\right)>0$ leads to $P J \cap P_{d}^{1} J \neq 0$, this is Hypothesis 1 of Lemma 1 .

Hypotheses 3 and 4 of Lemma 1 are clearly satisfied. The subspace of $\otimes^{n} H$ spanned by the characteristic functions of "rectangles" is dense in $\otimes^{n} H$; this is a property of product measures. Since the measure is continuous, the subspace of $\otimes^{n} H$ spanned by the characteristic functions of "rectangles" with disjoint sides is dense in $\otimes^{n} H$; any such characteristic function can be written as the sum of at most countably many functions of the same type, each of which is in the range of $P_{q}$ for some $q \in Q$.

To prove irreducibility, it obviously suffices to prove that Hypothesis 6 is satisfied since Hypotheses 7 and 8 can be proved in an identical manner.

We do this by using Lemma 1. In Lemma 1, let $J=P_{n}(D) H_{n y}, \Gamma=$ $R_{n y}\left(\left\{g \in G: R_{n y}(g) J=J\right\}\right) \mid J$ and $P=T_{n}(D)$. Let $Q$ be the set of those decompositions of $(X, \tau, m)$ which are subordinate to $D$. If $q \in Q$, let $P_{q}=P_{q}^{1}=T_{n}(q)$. The first four hypotheses of Lemma 1 are clearly satisfied since $P J \subseteq P_{q}^{1} J$ for each $q \in Q$. Hypothesis 5 follows from the definition of $P_{n}(D)$.

$T_{n}(D) H_{n y}$ is spanned by functions of the form

$$
\Omega\left(C\left(E_{1}\right) \otimes C\left(E_{2}\right) \otimes \cdots \otimes C\left(E_{n}\right)\right),
$$

where $\Omega$ is the projection of $\otimes^{n} H$ onto $H_{n y}, E_{i} \in D, 1 \leqq i \leqq n$, and $E_{i} \neq E_{j}$ if $i \neq j . \quad\{g \in G: g(E) \in D$ if $E \in D\}$ acts on $T_{n}(D) H_{n y}$ as an infinite permutation group; by [3, Lemma 5, part 3] this action is irreducible. Consequently, Hypothesis 6 of Lemma 1 is satisfied. Hypotheses 7 and 8 can be proved in an idential manner.

We now show that $\otimes^{n} R$ is disjoint from $\otimes^{m} R$ if $n>m$. Let the irreducible subrepresentation $R_{n y}\left(R_{m k}\right)$ of $\boldsymbol{\otimes}^{n} R\left(\otimes^{m} R\right)$ act on $H_{n}\left(H_{m}\right)$. Let $P_{n}\left(P_{m}\right)$ be the orthogonal projection with domain $\otimes^{n} H\left(\otimes^{m} H\right)$ and range $H_{n}\left(H_{m}\right)$.

Let $E_{i} \in D, 1 \leqq i \leqq n$, with $E_{i} \neq E_{j}$ if $i \neq j . \quad$ Let $v=C\left(E_{1}\right) \otimes$ 
$C\left(E_{2}\right) \otimes \cdots \otimes C\left(E_{n}\right) . \quad P_{n} v \neq 0$, and $R_{n y}(g) P_{n} v=P_{n} v$ if $g\left(E_{i}\right)=E_{i}$ for $1 \leqq i \leqq n$. Assume $U$ implements an equivalence between $R_{n y}$ and $R_{m k}$. Then $U P_{n} v$ is contained in the subspace of $H_{m}$ spanned by vectors of the form $P_{m} w$, where $w=f_{1} \otimes f_{2} \otimes \cdots \otimes f_{m}$ and $\left\{f_{i}: 1 \leqq i \leqq m\right\} \varsubsetneqq$ $\left\{C\left(E_{i}\right): 1 \leqq i \leqq n\right\}$. Assume $\left\{f_{i}: 1 \leqq i \leqq m\right\}=\left\{C\left(E_{i}\right): i \in s\right\}$, where $s$ is some proper subset of $\{1,2, \cdots, n\}$. Then $R_{m k}(g) P_{m} w=P_{m} w$ if $g\left(E_{i}\right)=E_{i}$ for $i \in s$. Consequently, $U^{-1} P_{m} w$ is in the subspace of $H_{n}$ spanned by vectors of the form $P_{n} v_{1}$, where $v_{1}=h_{1} \otimes h_{2} \otimes \cdots \otimes h_{n}$ and $\left\{h_{i}: 1 \leqq i \leqq n\right\} \subseteq$ $\left\{C\left(E_{i}\right): i \in s\right\}$. Then $v_{1} \perp v$, so that $U^{-1} U v \perp v$, which is a contradiction. The equivalence of $R_{n y}$ with $R_{n k}$ when and only when $y$ and $k$ correspond to the same partition of $n$ is a consequence of the properties of the regular representation of $S_{n}$ [9] and of the properties under equivalence of the set of common eigenvectors of eigenvalue 1 of a subset of a group.

Remarks. 1. Let $G$ have the weakest topology such that $R$ is weakly continuous. $G$ is a topological group in this topology but is not locally compact. $\otimes^{n} R$ is continuous for $n \in N$.

Let $G_{f}=\{g \in G|\exists E \in \tau: m(E)<\infty, g| X-E=I \mid X-E\}$, where $I$ is the identity map. $G_{f}$ is a dense subgroup of $G$. Consequently, the restriction of $\otimes^{n} R$ to the subgroup $G_{f}$ is irreducible.

2. Assume $(X, \tau, m)=\bigoplus_{\lambda \in \Lambda}\left(X_{\lambda}, \tau_{\lambda}, m_{\lambda}\right)$, where $\bigoplus$ means measure space direct sum [6] and $\left(X_{\lambda}, \tau_{\lambda}, m_{\lambda}\right)$ is a homogeneous measure space. Assume that $\left(X_{\lambda_{1}}, \tau_{\lambda_{1}}, m_{\lambda_{1}}\right) \oplus\left(X_{\lambda_{2}}, \tau_{\lambda_{2}}, m_{\lambda_{2}}\right)$ is not homogeneous if $\lambda_{1}, \lambda_{2} \in$ $\Lambda$ and $\lambda_{1} \neq \lambda_{2}$. Then $G=\Pi_{\lambda \in \Lambda} G_{\lambda}$, where $G\left(\operatorname{resp} . G_{\lambda}\right)$ is the automorphism group of the measure algebra of $(X, \tau, m)\left(\operatorname{resp} .\left(X_{\lambda}, \tau_{\lambda}, m_{\lambda}\right)\right)$, and $\Pi$ denotes complete direct product.

3. D. Maharam [4] has characterized continuous homogeneous measure spaces and has shown that any $\sigma$-finite continuous measure space is the measure space direct sum of homogeneous measure spaces.

4. A continuous measure space is pointwise homogeneous if and onlf if two principal ideals of its measure algebra are isomorphic via a measure preserving transformation whenever their generators have equal and finite measure. If $(X, \tau, m)$ is an infinite continuous pointwise homogeneous measure space, then the restriction of $R_{n y}$ to the group of measure preserving transformations of $(X, \tau, m)$ is irreducible. The proof is identical to the proof of Theorem 1 .

5. The theorem is not valid if $m$ is a finite continuous homogeneous measure since then $G$ acts irreducibly on the constant functions in $\otimes^{n} H$. The author does not know if the theorem holds with modifications. The difficulty is that Lemma 2 is false for finite measures.

6. If the measure $m$ is not $\sigma$-finite, it is possible in the proof 
of the theorem that $m\left(F_{2} \cap F_{3}\right)=0$ for all $F_{2} \in d$ and $F_{3} \in D$. This is the reason for the complexity of Lemma 1.

Let $I$ be the unit interval. Let $(I, \tau, m)$ be the unit interval with Lebesgue measure; let $\left(I, \tau_{1}, m_{1}\right)$ be the unit interval with $\tau_{1}$ the $\sigma$-algebra generated by all finite subsets of $I$ and $m_{1}$ the counting measure. Let $B$ be the measure algebra of $(I, \tau, m) \times\left(I, \tau_{1}, m_{1}\right)$.

Consider the measure space $\left(I \times I, \tau_{2}, m_{2}\right)$, where $\tau_{2}$ is the $\sigma$-algebra generated by sets of the form $b_{1} \times E_{1}$ and $E_{2} \times b_{2}$, with $b_{1}, b_{2} \in I, E_{1}, E_{2} \in$ $\tau, m_{2}\left(b_{1} \times E_{1}\right)=m_{1}\left(E_{1}\right)$, and $m_{2}\left(E_{2} \times b_{2}\right)=m_{1}\left(E_{2}\right)$; let $A$ be the measure algebra of $\left(I \times I, \tau_{2}, m_{2}\right)$.

$A$ is a continuous homogeneous measure algebra; any principle ideal of $A$ whose generator has finite measure $c$ is isomorphic to the measure algebra of the interval $[0, c]$. Note that $A$ is isomorphic to $B \oplus B$.

Let $D_{1}=\{b \times I: b \in I\}$ and $D_{2}=\{I \times b: b \in I\} . \quad D_{1}$ and $D_{2}$ are decompositions of $\left(I \times I, \tau_{2}, m_{2}\right)$ of type 1. $m_{2}\left(\left(b_{1} \times I\right) \cap\left(I \times b_{2}\right)\right)=0$ for all $b_{1}, b_{2} \in I$.

\section{REFERENCES}

1. J. Dixmier, Les Algèbres d'Opérateurs dans l'Espace Hilbertien, Gauthier-Villars, Paris, 1957.

2. Michael Keane, Contractibility of the automorphism group of a nonatomic measure space, Proc. Amer. Math. Soc., 26 (1970), 420-422.

3. A. L. Lieberman, The structure of certain unitary representations of infinite symmetric groups, Trans. Amer. Math. Soc., 164 (1970), 189-198.

4. D. Maharam, On Homogeneous Measure Algebras, PNAS, 28 (1942), 108-111.

5. H. L. Royden, Real Analysis, MacMillan Company, New York, 1963.

6. I. E. Segal, Equivalences of measure spaces, Amer. J. Math., 73 (1951), 275-313.

7. - Tensor Algebras over Hilbert Spaces, I, Trans. Amer. Math. Soc., 81 (1956), 106-134.

8. - The structure of a class of representations of the unitary group on a Hilbert space, Proc. Amer. Math. Soc., 8 (1957), 197-203.

9. H. Weyl, The Classical Groups, Princeton University Press, Princeton, 1946.

Received April 19, 1971.

UNIVERSITY OF SOUTH FLORIDA 


\section{PACIFIC JOURNAL OF MATHEMATICS}

\section{EDITORS}

\section{H. SAMELSON}

Stanford University

Stanford, California 94305

C. R. Новву

University of Washington Seattle, Washington 98105

\section{J. DuGundJI}

Department of Mathematics University of Southern California Los Angeles, California 90007

RICHARD ARENS

University of California Los Angeles, California 90024

\section{ASSOCIATE EDITORS}
E. F. BECKENBACH
B. H. NeumanN
F. WOLF
K. YoSHIDA

\section{SUPPORTING INSTITUTIONS}

\author{
UNIVERSITY OF BRITISH COLUMBIA \\ CALIFORNIA INSTITUTE OF TECHNOLOGY \\ UNIVERSITY OF CALIFORNIA \\ MONTANA STATE UNIVERSITY \\ UNIVERSITY OF NEVADA \\ NEW MEXICO STATE UNIVERSITY \\ OREGON STATE UNIVERSITY \\ UNIVERSITY OF OREGON \\ OSAKA UNIVERSITY
}

\author{
UNIVERSITY OF SOUTHERN CALIFORNIA \\ STANFORD UNIVERSITY \\ UNIVERSITY OF TOKYO \\ UNIVERSITY OF UTAH \\ WASHINGTON STATE UNIVERSITY \\ UNIVERSITY OF WASHINGTON \\ $*{ }^{*}$
AMERICAN MATHEMATICAL SOCIETY
NAVAL WEAPONS CENTER
}

The Supporting Institutions listed above contribute to the cost of publication of this Journal, but they are not owners or publishers and have no responsibility for its content or policies.

Mathematical papers intended for publication in the Pacific Journal of Mathematics should be in typed form or offset-reproduced, (not dittoed), double spaced with large margins. Underline Greek letters in red, German in green, and script in blue. The first paragraph or two must be capable of being used separately as a synopsis of the entire paper. The editorial "we" must not be used in the synopsis, and items of the bibliography should not be cited there unless absolutely necessary, in which case they must be identified by author and Journal, rather than by item number. Manuscripts, in duplicate if possible, may be sent to any one of the four editors. Please classify according to the scheme of Math. Rev. Index to Vol, 39. All other communications to the editors should be addressed to the managing editor, Richard Arens, University of California, Los Angeles, California, 90024.

50 reprints are provided free for each article; additional copies may be obtained at cost in multiples of 50 .

The Pacific Journal of Mathematics is issued monthly as of January 1966. Regular subscription rate: $\$ 48.00$ a year (6 Vols., 12 issues). Special rate: $\$ 24.00$ a year to individual members of supporting institutions.

Subscriptions, orders for back numbers, and changes of address should be sent to Pacific Journal of Mathematics, 103 Highland Boulevard, Berkeley, California, 94708.

PUBLISHED BY PACIFIC JOURNAL OF MATHEMATICS, A NON-PROFIT CORPORATION

Printed at Kokusai Bunken Insatsusha (International Academic Printing Co., Ltd.), 270, 3-chome Totsuka-cho, Shinjuku-ku, Tokyo 160, Japan. 


\section{Pacific Journal of Mathematics}

\section{Vol. 44, No. $2 \quad$ June, 1973}

Tsuyoshi Andô, Closed range theorems for convex sets and linear liftings . . . . . . 393

Richard David Bourgin, Conically bounded sets in Banach spaces . . . . . . . . . 411

Robert Jay Buck, Hausdorff dimensions for compact sets in $R^{n} \ldots \ldots \ldots \ldots \ldots \ldots . \ldots 421$

Henry Cheng, A constructive Riemann mapping theorem ................ 435

David Fleming Dawson, Summability of subsequences and stretchings of

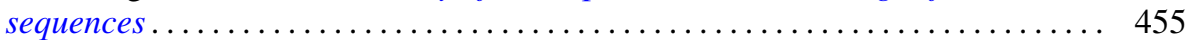

William Thomas Eaton, A two sided approximation theorem for 2-spheres ....... 461

Jay Paul Fillmore and John Herman Scheuneman, Fundamental groups of compact complete locally affine complex surfaces ....................... 487

Avner Friedman, Bounded entire solutions of elliptic equations . . . . . . . . . . . 497

Ronald Francis Gariepy, Multiplicity and the area of an $(n-1)$ continuous

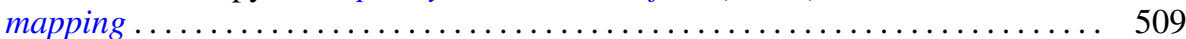

Andrew M. W. Glass, Archimedean extensions of directed interpolation groups . . . . 515

Morisuke Hasumi, Extreme points and unicity of extremum problems in $H^{1}$ on

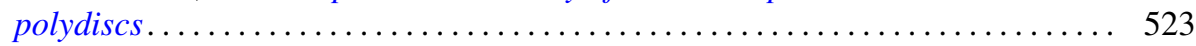

Trevor Ongley Hawkes, On the Fitting length of a soluble linear group . . . . . . 537

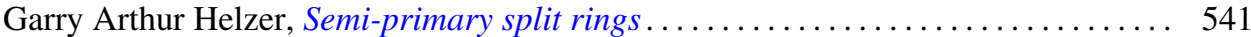

Melvin Hochster, Expanded radical ideals and semiregular ideals . . . . . . . . . 553

Keizō Kikuchi, Starlike and convex mappings in several complex variables . . . . . . 569

Charles Philip Lanski, On the relationship of a ring and the subring generated by its

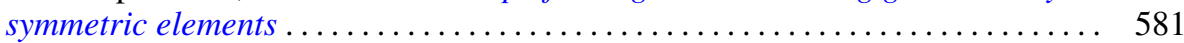

Jimmie Don Lawson, Intrinsic topologies in topological lattices and semilattices ........................................... 593

Roy Bruce Levow, Counterexamples to conjectures of Ryser and de Oliveira ...... 603

Arthur Larry Lieberman, Some representations of the automorphism group of an infinite continuous homogeneous measure algebra ..........

William George McArthur, $G_{\delta}$-diagonals and metrization theorems $\ldots .$.

James Murdoch McPherson, Wild arcs in three-space. II. An invariant of

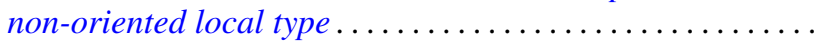

H. Millington and Maurice Sion, Inverse systems of group-valued measures ...

C. Edward Moore, Concrete semispaces and lexicographic separation of convex

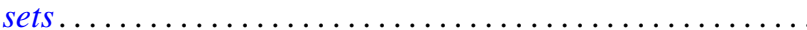

Jingyal Pak, Actions of torus $T^{n}$ on $(n+1)$-manifolds $M^{n+1}$.

Merrell Lee Patrick, Extensions of inequalities of the Laguerre and Turán type . . . . 675

Harold L. Peterson, Jr., Discontinuous characters and subgroups of finite index. . . . 683

S. P. Philipp, Abel summability of conjugate integrals . . . . . . . . . . . . . 693

R. B. Quintana and Charles R. B. Wright, On groups of exponent four satisfying an

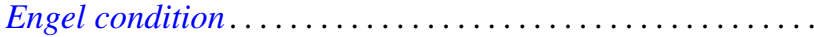

Marlon C. Rayburn, On Hausdorff compactifications. . . . . . . . . .

Martin G. Ribe, Necessary convexity conditions for the Hahn-Banach theorem in

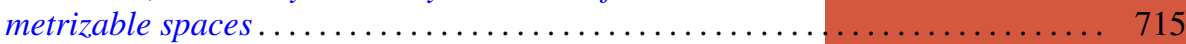

Ryōtarō Satō, On decomposition of transformations in infinite measure spaces .... 733

Peter Drummond Taylor, Subgradients of a convex function obtained from a

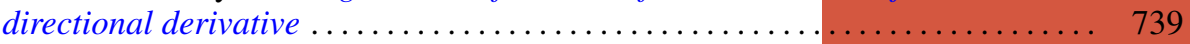

James William Thomas, A bifurcation theorem for $k$-set contractions . . . . . . . . 749 Clifford Edward Weil, A topological lemma and applications to real functions . . . . 757

Stephen Andrew Williams, A nonlinear elliptic boundary value problem . . ....... 767

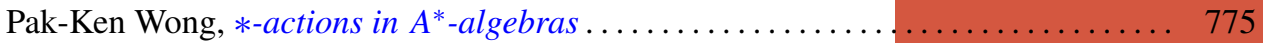

